\title{
Relativistic and Quantum Revisions of the Multisource Thermal Model in High-Energy Collisions
}

\author{
Fu-Hu Liu, Cai-Xing Tian, Mai-Ying Duan, \\ and Bao-Chun Li
}

Institute of Theoretical Physics, Shanxi University, Shanxi, Taiyuan 030006, China

Correspondence should be addressed to Fu-Hu Liu, fuhuliu@163.com

Received 28 July 2012; Accepted 19 October 2012

Academic Editor: Terry Sloan

Copyright (c) $2012 \mathrm{Fu}-\mathrm{Hu}$ Liu et al. This is an open access article distributed under the Creative Commons Attribution License, which permits unrestricted use, distribution, and reproduction in any medium, provided the original work is properly cited.

The multisource thermal model is revised for relativistic and quantum situations. It is shown that the quantum effect can be neglected due to a very small result. The distributions of particle momenta, momentum components, transverse momenta, kinetic energies, and velocities in both the classical and relativistic situations are presented to give comparisons.

\section{Introduction}

The multisource thermal model (or previously the multisource ideal gas model) was suggested by us in the past years [1-4]. This model assumes that many emission sources of produced particles and nuclear fragments are formed in high-energy collisions. Each emission source is treated as a thermal equilibrium system of classical ideal gas. Then, the classical Maxwell's ideal gas model and corresponding distributions [5] can be used in the multisource thermal model. Because the classical Maxwell's ideal gas model has no consideration of the relativistic and quantum effects, some revisions are needed when we consider the model's application in high-energy collisions.

In this paper, the relativistic and quantum effects are considered for each emission source. The multisource thermal model [1-4] is then revised for the relativistic and quantum situations. Distributions of particle momenta, momentum components, transverse momenta, kinetic energies, and velocities in both the classical and relativistic situations are given and compared. 


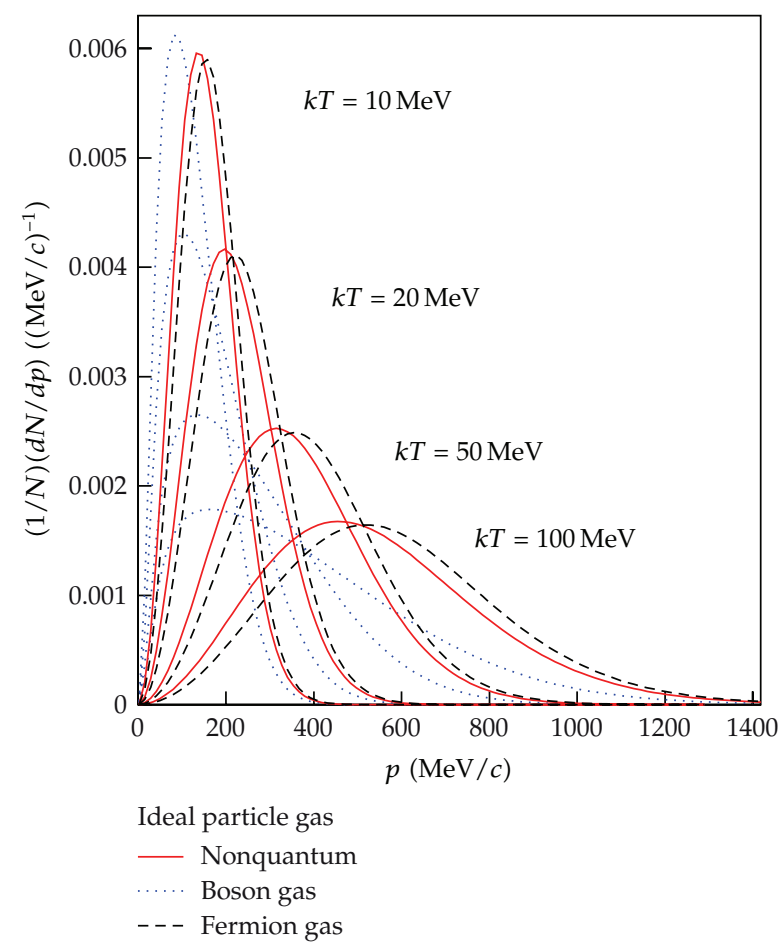

Figure 1: Momentum distributions of relativistic nonquantum particles (solid curves), bosons (dotted curves), and fermions (dashed curves) in ideal gas model calculated by (2.6) and (2.7), respectively, with $m_{0}=938 \mathrm{MeV} / c^{2}$ and $\mu=937 \mathrm{MeV}$. The results are given for four temperatures indicated in the figure.

\section{Formulations and Comparisons}

Let $k$ and $T$ denote the Boltzmann constant and temperature of thermal equilibrium system, respectively, and $m_{0}, p, p_{x, y, z}, p_{T}, E_{K}, v, \theta$, and $\varphi$ denote the rest mass, momentum, momentum components, transverse momentum, kinetic energy, velocity, emission angle, and azimuth of a concerned particle, respectively. In our discussions, we define the beam direction to be the $o z$ axis and the reaction plane to be the $x o z$ plane in high-energy collisions.

In the classical Maxwell's ideal gas model [5], we have the distributions of particle momenta, momentum components, transverse momenta, kinetic energies, and velocities to be

$$
\begin{gathered}
f_{p}(p)=\frac{1}{N} \frac{d N}{d p}=\sqrt{\frac{2}{\pi}} \frac{p^{2}}{\left(m_{0} k T\right)^{3 / 2}} \exp \left(-\frac{p^{2}}{2 m_{0} k T}\right), \\
f_{p_{x, y, z}}\left(p_{x, y, z}\right)=\frac{1}{N} \frac{d N}{d p_{x, y, z}}=\frac{1}{\sqrt{2 \pi} \sqrt{m_{0} k T}} \exp \left(-\frac{p_{x, y, z}^{2}}{2 m_{0} k T}\right), \\
f_{p_{T}}\left(p_{T}\right)=\frac{1}{N} \frac{d N}{d p_{T}}=\frac{p_{T}}{m_{0} k T} \exp \left(-\frac{p_{T}^{2}}{2 m_{0} k T}\right),
\end{gathered}
$$




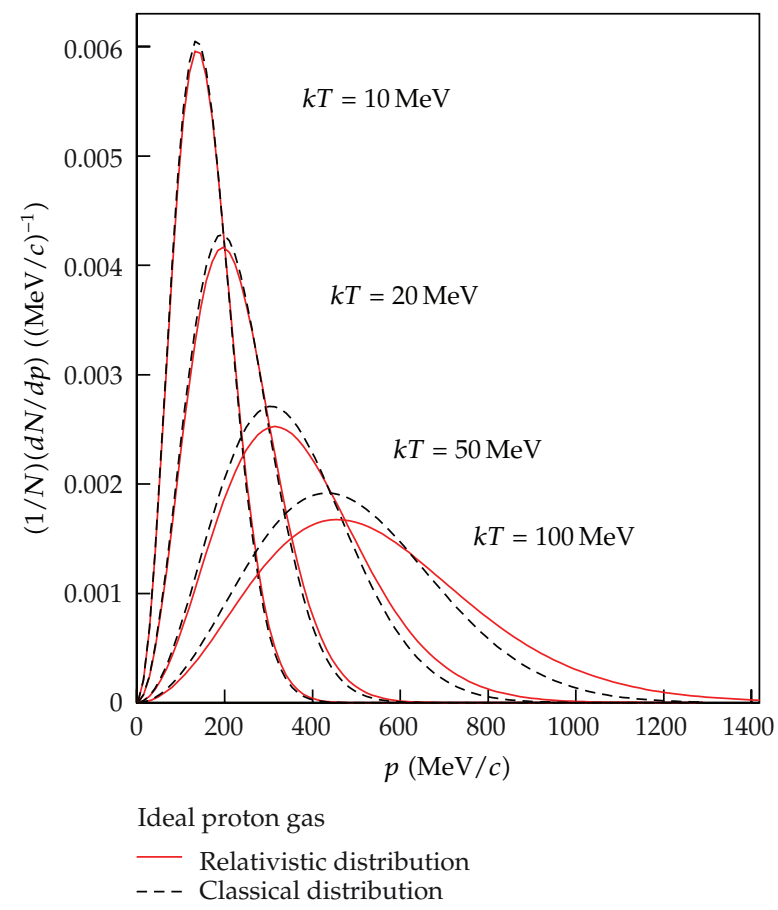

Figure 2: Proton momentum distributions in relativistic (nonquantum) ideal gas model (solid curves) and classical ideal gas model (dashed curves). The different curves are calculated by (2.6) and (2.1), respectively, and are given for four temperatures indicated in the figure. The solid curves in Figure 2 are the same as those in Figure 1.

$$
\begin{gathered}
f_{E_{K}}\left(E_{K}\right)=\frac{1}{N} \frac{d N}{d E_{K}}=\frac{2}{\sqrt{\pi}}\left(\frac{1}{k T}\right)^{3 / 2} \sqrt{E_{K}} \exp \left(-\frac{E_{K}}{k T}\right), \\
f_{v}(v)=\frac{1}{N} \frac{d N}{d v}=\sqrt{\frac{2}{\pi}}\left(\frac{m_{0}}{k T}\right)^{3 / 2} v^{2} \exp \left(-\frac{m_{0} v^{2}}{2 k T}\right),
\end{gathered}
$$

respectively.

Considering the relativistic effect [6,7], we have

$$
f_{p}(p)=\frac{1}{N} \frac{d N}{d p}=C p^{2} \exp \left(-\frac{\sqrt{p^{2}+m_{0}^{2}}}{k T}\right)
$$

where $C=\left(1 / m_{0}^{2} k T\right)\left(1 / K_{2}\left(m_{0} / k T\right)\right)$ is the normalization constant and $K_{2}\left(m_{0} / k T\right)$ is the modified Bessel function of order 2 . In the above equation, we have taken the natural unit 


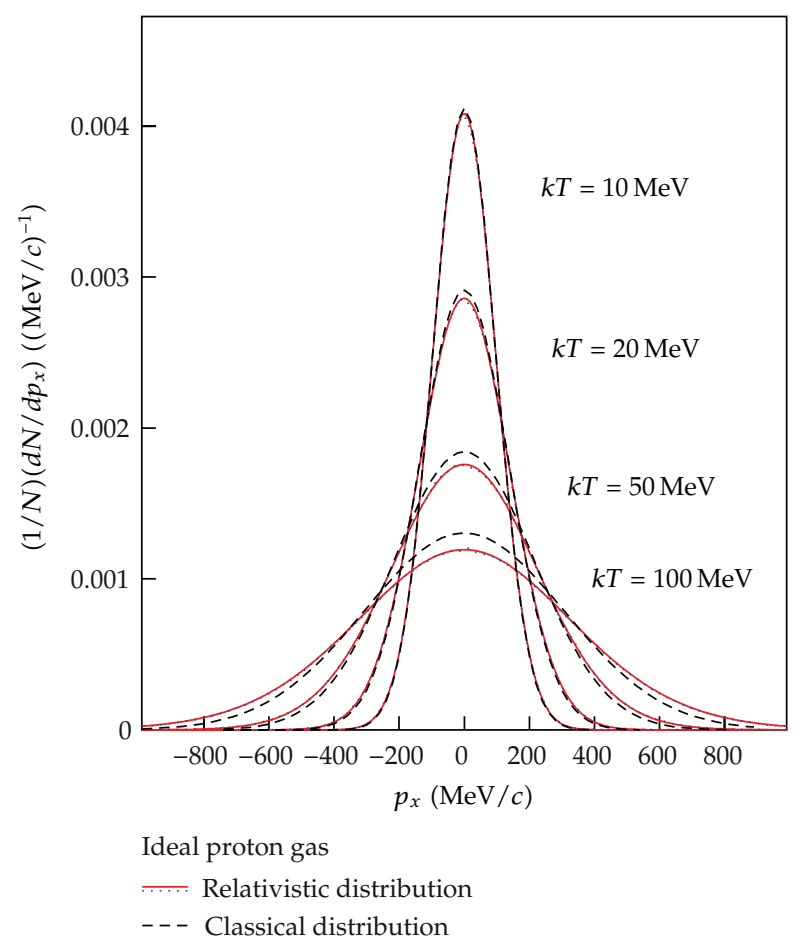

Figure 3: Proton momentum component distributions in relativistic ideal gas model (solid and dotted curves) and classical ideal gas model (dashed curves). The different curves are calculated by (2.8), Monte Carlo method, and (2.2), respectively, and are given for four temperatures indicated in the figure.

system in which the speed $c$ of light in vacuum is 1 . Considering the quantum effect [8], we have

$$
f_{p}(p)=\frac{1}{N} \frac{d N}{d p}=C_{1} p^{2}\left[\exp \left(\frac{\sqrt{p^{2}+m_{0}^{2}}-\mu}{k T}\right) \pm 1\right]^{-1}
$$

where $C_{1}$ is the normalization constant, $\mu$ is the chemical potential, +1 denotes fermions, and -1 denotes bosons, respectively. Our calculations show that, comparing with (2.6), the quantum effect in (2.7) with $\mu$ having large dispersion from $m_{0}$ is so small that we do not need to consider it in studying momentum distributions in high-energy collisions. The chemical potential also causes a very small effect in the case of neglecting \pm 1 . Only the combination of quantum effect and $\mu \approx m_{0}$ causes an obvious dispersion from (2.6). As an example, by taking $m_{0}=938 \mathrm{MeV} / c^{2}$ and $\mu=937 \mathrm{MeV}$, the calculated momentum distributions for relativistic nonquantum gas (see (2.6)), boson gas (see (2.7) with -1), and fermion gas (see (2.7) with +1 ) at $k T=10,20,50$, and $100 \mathrm{MeV}$ are shown in Figure 1 by the solid, dotted, and dashed curves, respectively. One can see obvious differences in momentum distributions among the three kinds of ideal gases. Especially at higher temperature, this difference is more obvious.

As the relativistic and quantum revisions of (2.1), (2.7) can be used in the multisource thermal model. To avoid the unbending choice of $\mu$ and \pm 1 , in the following we use (2.6) to give comparisons with (2.1)-(2.5). In Figure 2, the particle momentum distributions 


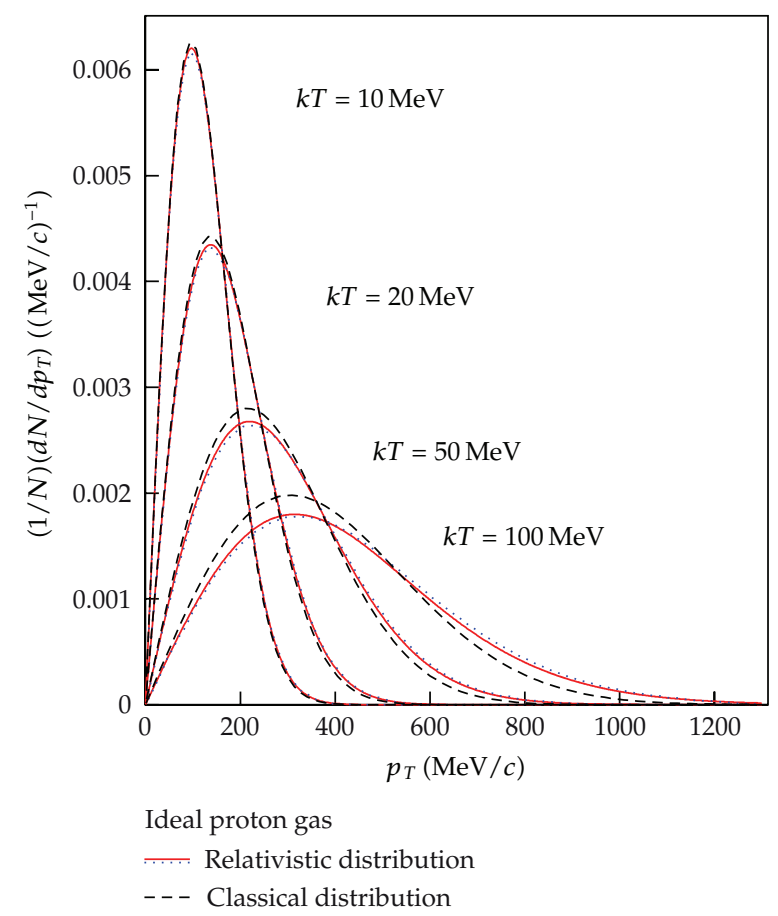

Figure 4: Proton transverse momentum distributions in relativistic ideal gas model (solid and dotted curves) and classical ideal gas model (dashed curves). The different curves are calculated by (2.9), Monte Carlo method, and (2.3), respectively, and are given for four temperatures indicated in the figure.

at $k T=10,20,50$, and $100 \mathrm{MeV}$ calculated by (2.6) and (2.1) are given by the solid and dashed curves, respectively. In the calculation, we take $m_{0}=938 \mathrm{MeV} / c^{2}$ which means that an ideal proton gas is considered. One can see that the difference between the classical distribution and relativistic one is not obvious at lower temperatures (10 and $20 \mathrm{MeV})$, while the difference is obvious at higher temperatures (50 and $100 \mathrm{MeV})$.

The momentum components can be given by $p_{x}=p \sin \theta \cos \varphi, p_{y}=p \sin \theta \sin \varphi$, and $p_{z}=p \cos \theta$, respectively. An isotropic ideal gas model gives the same distribution of three different components, and the emission angular and azimuthal distributions are $f_{\theta}(\theta)=$ $(1 / 2) \sin \theta$ and $f_{\varphi}(\varphi)=1 / 2 \pi$, respectively. Combining with (2.6), we can use the Monte Carlo method and analytical expression to give the momentum component distributions at relativistic energy. In Figure 3, the dashed curve represents the classical distribution (see (2.2)) of $p_{x}$, and the dotted and solid curves represent the relativistic distributions by using the Monte Carlo method and analytical expression

$$
f_{p_{x}}\left(p_{x}\right)=\frac{1}{N} \frac{d N}{d p_{x}}=C_{2} \sqrt{p_{x}^{2}+m_{0}^{2}} \exp \left(-\frac{\sqrt{p_{x}^{2}+m_{0}^{2}}}{k T}\right)
$$

respectively, where $C_{2}$ is the normalization constant. All the distributions are given at four temperatures mentioned in the figure. We see that the difference between the classical and relativistic distributions at lower temperature is not obvious, and the difference at higher 


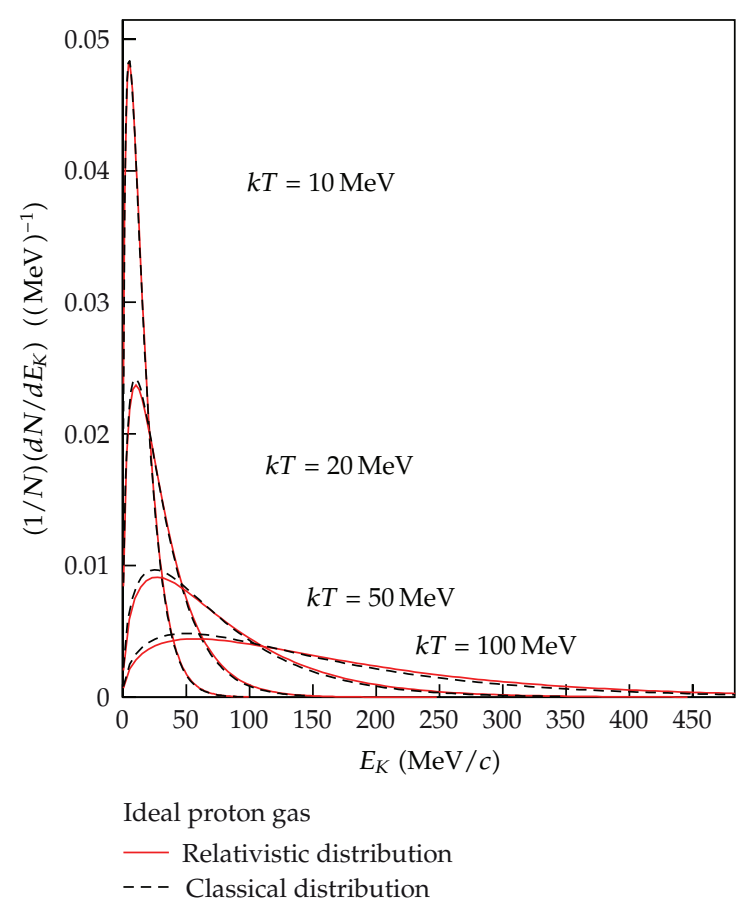

Figure 5: Proton kinetic energy distributions in relativistic ideal gas model (solid curves) and classical ideal gas model (dashed curves). The different curves are calculated by (2.10) and (2.4), respectively, and are given for four temperatures indicated in the figure.

temperature is obvious. The relativistic distributions obtained by the Monte Carlo method and analytical expression are almost the same. This result confirms the two methods all together.

The transverse momentum is given by $p_{T}=p \sin \theta$. Combining with (2.6), we can use the Monte Carlo method and analytical expression to give the transverse momentum distribution at relativistic energy. In Figure 4, the dashed curve represents the classical distribution (see (2.3)) of $p_{T}$, whereas the dotted and solid curves represent the relativistic results by using the Monte Carlo method and Boltzmann distribution

$$
f_{p_{T}}\left(p_{T}\right)=\frac{1}{N} \frac{d N}{d p_{T}}=C_{3} p_{T} \exp \left(-\frac{\sqrt{p_{T}^{2}+m_{0}^{2}}}{k T}\right)
$$

respectively, where $C_{3}$ is the normalization constant. All the distributions are given at four temperatures. We see again that the difference between the classical distribution and relativistic one is small at lower temperature and large at higher temperature. The relativistic results obtained by the Monte Carlo method and Boltzmann distribution are almost the same. This confirms the two methods all together. 


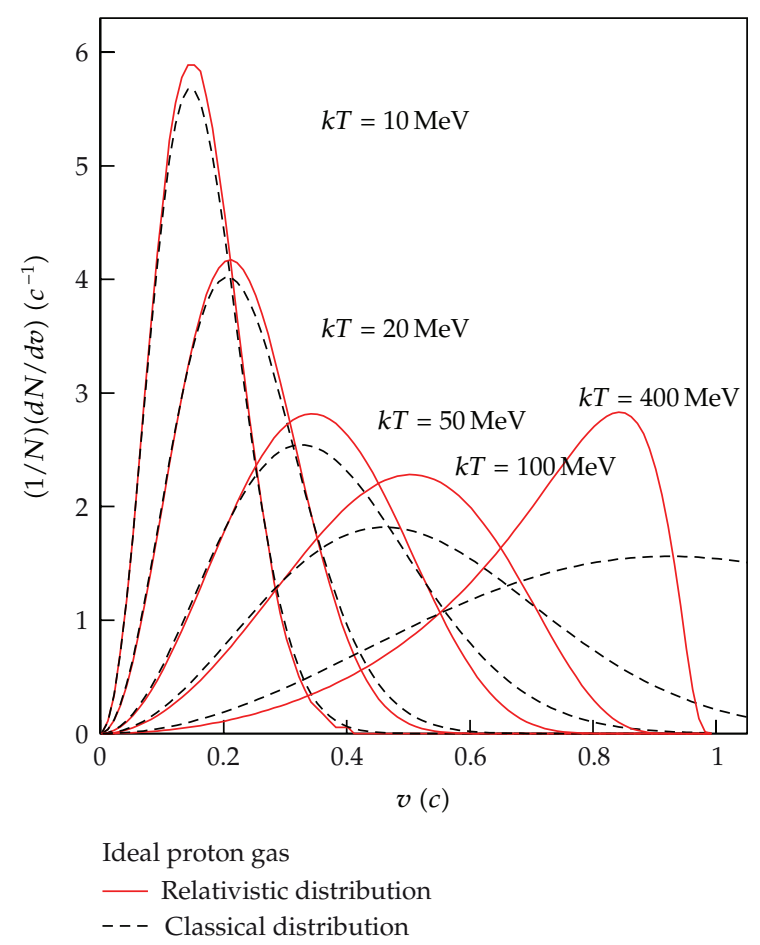

Figure 6: Proton velocity distributions in relativistic ideal gas model (solid curves) and classical ideal gas model (dashed curves). The different curves are calculated by (2.12) and (2.5), respectively, and are given for five temperatures indicated in the figure.

The kinetic energy in relativistic situation can be given by $E_{K}=\sqrt{p^{2}+m_{0}^{2}}-m_{0}$; combining with (2.6), we have the distribution of kinetic energy in relativistic situation to be

$$
f_{E_{K}}\left(E_{K}\right)=\frac{1}{N} \frac{d N}{d E_{K}}=C\left(E_{K}+m_{0}\right) \sqrt{\left(E_{K}+m_{0}\right)^{2}-m_{0}^{2}} \exp \left(-\frac{E_{K}+m_{0}}{k T}\right) .
$$

The total energy $E=\sqrt{p^{2}+m_{0}^{2}}=E_{K}+m_{0}$ is simply given by

$$
f_{E}(E)=\frac{1}{N} \frac{d N}{d E}=C E \sqrt{E^{2}-m_{0}^{2}} \exp \left(-\frac{E}{k T}\right) .
$$

For ideal gas in classical situation, the energy is only the kinetic energy due to zero potential energy. The comparisons between the kinetic energy distributions in classical situation (dashed curves) and relativistic case (solid curves) are presented in Figure 5. The two curves at lower temperature are almost the same, and those at higher temperature are very similar. 
The relationship between momentum and velocity at relativistic energy gives $p=$ $m_{0} v / \sqrt{1-v^{2}}$. Combining with (2.6), we have the distribution of velocity to be

$$
f_{v}(v)=\frac{1}{N} \frac{d N}{d v}=C \frac{m_{0}^{3} v^{2}}{\left(1-v^{2}\right)^{5 / 2}} \exp \left(-\frac{m_{0}}{k T \sqrt{1-v^{2}}}\right)
$$

The comparisons between the velocity distributions in classical situation (dashed curves) and relativistic case (solid curves) are presented in Figure 6. There are small differences at lower temperatures and large differences at higher temperatures. One sees $v>c$ in classical situation at around $100 \mathrm{MeV}$. Especially, the probability of particles with $v>c$ is very large at $400 \mathrm{MeV}$. Obviously, the relativistic effect has to be considered at these higher temperatures.

\section{Conclusion}

In conclusion, the multisource thermal model [1-4] is revised in this paper for relativistic and quantum situations. The quantum effect in particle momentum distributions in highenergy collisions is very small when the chemical potential has a large dispersion from the rest mass of the concerned particle. The chemical potential also causes a very small effect when we neglect \pm 1 . Only the combination of quantum effect and nearly the same chemical potential and rest mass causes an obvious dispersion from the classical distribution. In the distributions of particle momenta, momentum components, transverse momenta, kinetic energies, and velocities, the relativistic effect is small at lower temperatures and large at higher temperatures. This is a natural result. Because of the present work, the classical distributions used in the multisource thermal model [1-4] can be replaced by the revised distributions.

\section{Acknowledgments}

This work was supported by the National Natural Science Foundation of China under Grant no. 10975095, the National Fundamental Fund of Personnel Training under Grant no. J1103210, the Open Research Subject of the Chinese Academy of Sciences Large-Scale Scientific Facility under Grant no. 2060205, and the Shanxi Scholarship Council of China.

\section{References}

[1] F. H. Liu, "Longitudinal and transverse flows of protons in 2-8 A GeV Au-Au collisions," Europhysics Letters, vol. 63, no. 2, pp. 193-199, 2003.

[2] F. H. Liu, N. N. Abd Allah, D. H. Zhang, and M. Y. Duan, "Angular distributions of target black fragments in nucleus-nucleus collisions at high energy," International Journal of Modern Physics E, vol. 12, no. 5, pp. 713-723, 2003.

[3] F. H. Liu, N. N. Abd Allah, and B. K. Singh, "Dependence of black fragment azimuthal and projected angular distributions on polar angle in silicon-emulsion collisions at $4.5 \mathrm{~A} \mathrm{GeV} / c$," Physical Review C, vol. 69, no. 5, Article ID 057601, 4 pages, 2004.

[4] F. H. Liu, J. S. Li, and M. Y. Duan, "Light fragment emission in ${ }^{86} \mathrm{Kr}^{124} \mathrm{Sn}$ collisions at $25 \mathrm{MeV} /$ nucleon," Physical Review C, vol. 75, no. 5, Article ID 054613, 5 pages, 2007.

[5] Y. H. Qin and Thermal Physics Higher Education Press, Beijing, China, 2011.

[6] J. L. Synge, The Relativistic Gas, North-Holland, Amsterdam, The Netherlands, 1957. 
[7] C. D. Dermer, "The production spectrum of a relativistic Maxwell-Boltzmann gas," The Astrophysical Journal, vol. 280, no. 1, pp. 328-333, 1984.

[8] P. Z. Ning, L. Li, and D. F. Min, Foundation of Nuclear Physics: Nucleons and Nuclei, Higher Education Press, Beijing, China, 2003. 

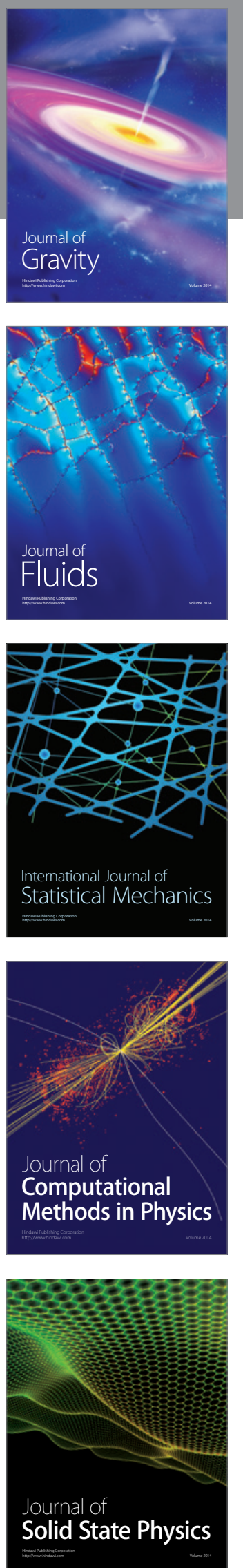

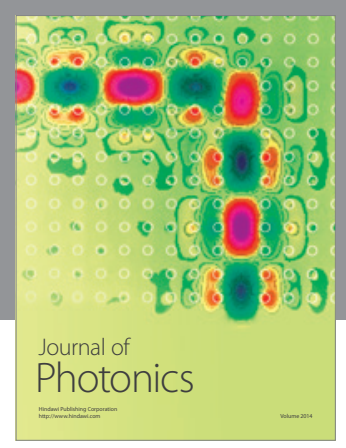

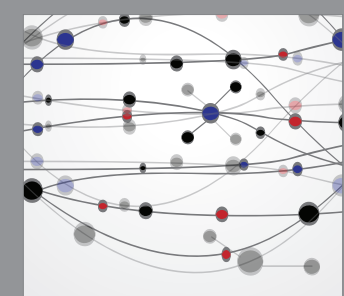

The Scientific World Journal
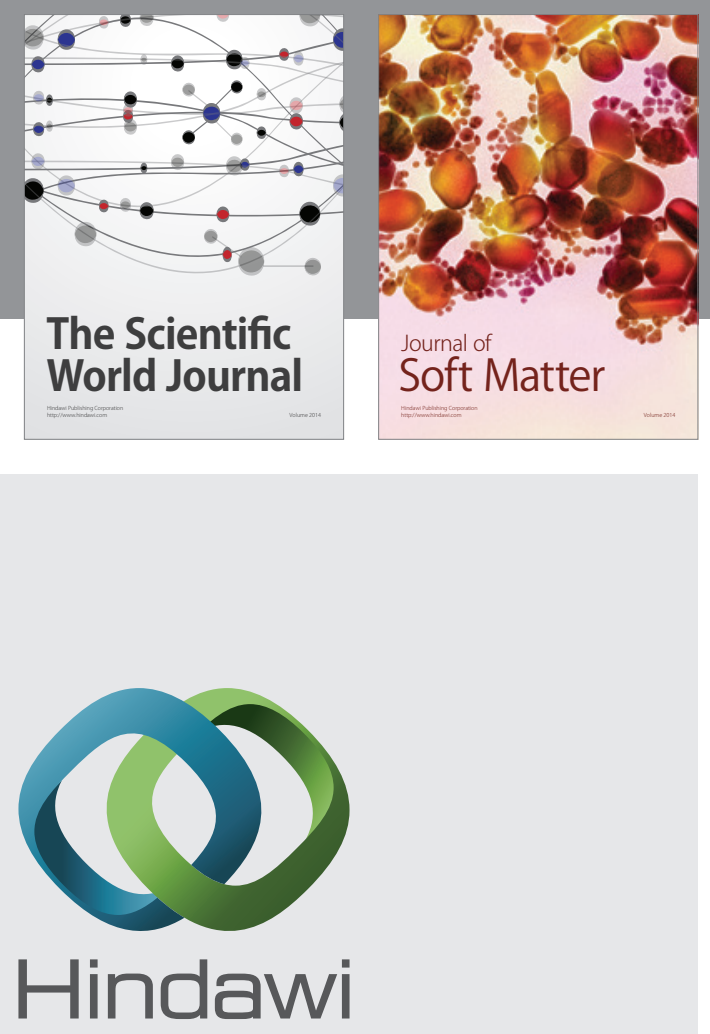

Submit your manuscripts at

http://www.hindawi.com
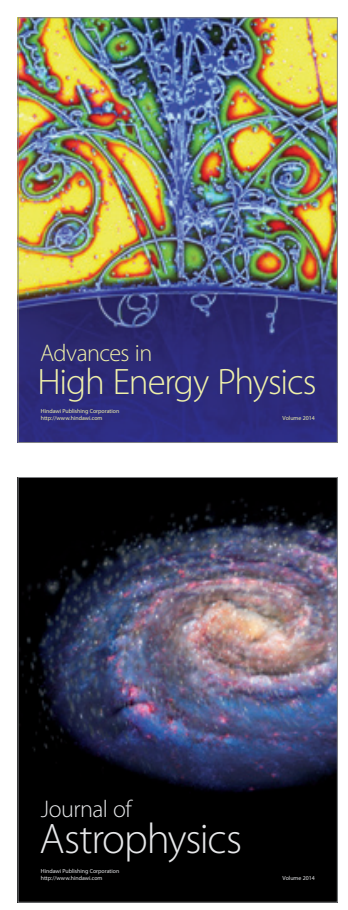
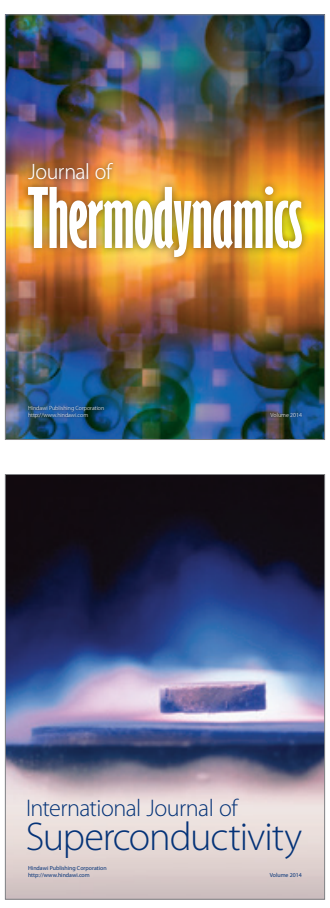
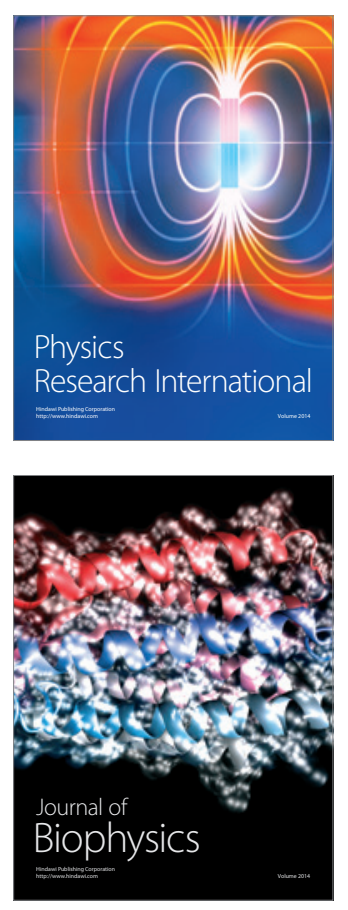
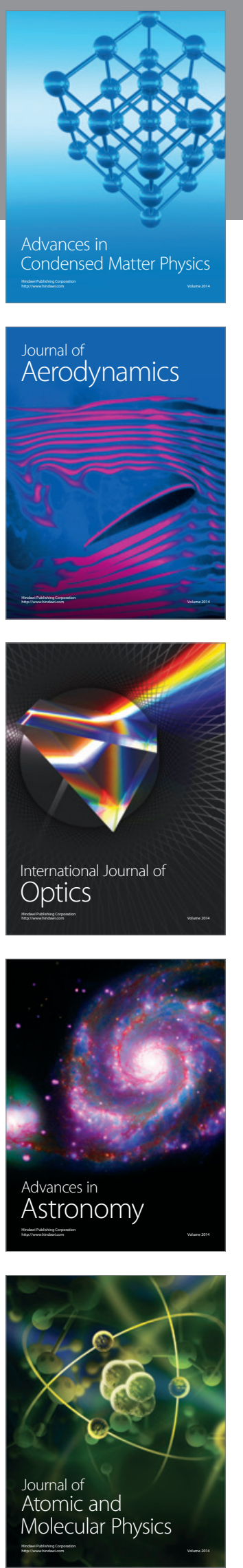